\title{
Keynote Talk: Tracking the Progress of Multimedia Semantics - from MPEG-7 to Web 3.0
}

\author{
Jane Hunter \\ The University of Queensland, Australia
}

Since 2001 when the original MPEG-7 standard was published, there have been a number of approaches to representing MPEG-7 as an ontology to enhance the semantic interoperability and richness of multimedia metadata. Subsequently range of tools/services, based on the MPEG-7 ontology, have been developed to generate both MPEG-7 and higher level semantic descriptions. In particular, a number of systems focused on semi-automatic semantic annotation of multimedia. These systems combine machine-learning techniques with manual ontologybased annotation to enhance the indexing and querying of multimedia content. However they depend on the availability of a training corpus of labelled multimedia content - which is difficult and expensive to generate. Most recently, there has been an explosion of social tagging tools on the Internet as part of the Web 2.0 phenomena. Such systems provide a community-driven approach to classifying resources on the Web, so that they can be browsed, discovered and re-used. Proponents of social tagging systems argue that they generate more relevant, light weight and cheaper metadata than traditional cataloguing systems. The future challenge is to leverage the potential of community-driven social tagging systems. This paper will describe the next generation of hybrid scalable classification systems that combine social tagging, machine-learning and traditional library classification approaches. It will also discuss approaches to the related challenge of aggregating light-weight community-generated tags with complex MPEG-7 descriptions and discipline-specific ontologies through common, extensible upper ontologies - to enhance discovery and re-use of multimedia content across disciplines and communities. 